\title{
Postkoloniale Perspektiven und Forschendes Lernen: Ein Beispiel aus der kirchengeschichtlichen Lehrpraxis
}

\author{
Sabine Hübner
}

\begin{abstract}
How can postcolonial, power-critical perspectives enrich seminars on the history of Christianity? In order to pursue this question, this contribution provides an example for the practice of teaching. The seminar used photographs from the North German Mission Society (19th and 2oth century) as a central source. In accordance with the didactic format of research-based learning, the participants carried out small-scale research projects with a special focus on the issues of visual representation and power. The aim of the analysis of the photographs was to shed light on the complexity of colonialism and Christian missions, and to increase awareness of the ongoing impact of this history. As elaborated in this article, postcolonial and research-based approaches mutually strengthen each other. On the one hand, postcolonial questions lead to a power-critical broadening of research-based teaching concepts. On the other hand, research-based seminars offer a suitable context in which students gain a better understanding of postcolonial reflections.
\end{abstract}

\section{Einleitung}

Ein Jugendlicher in einem hellen, dezent gestreiften Anzug steht neben einem Wiener Kaffeehausstuhl. Den rechten Arm stützt er elegant auf die Stuhllehne, in der linken Hand hält er ein Buch. Der Junge steht vor einer Tapete mit floralem Muster und ist barfuß.

Etwa 20 Schülerinnen sitzen an ihren Tischen und haben Bücher aufgeklappt vor sich liegen. Die Lehrerin sitzt schräg vor ihnen, im Hintergrund steht eine junge Mitarbeiterin. Neben der Klasse auf dem Boden befinden sich ein Rechenschieber zur Bearbeitung von mathematischen Aufgaben und eine große Tafel, auf der in großem und deutlichem Schriftbild Buchstaben aus der Ewe-Sprache zu sehen sind. 
Diese Szenen sind auf historischen Fotografien abgebildet, die zum Bildbestand der 1836 gegründeten Norddeutschen Mission (= NMG) gehören und heute als Depositum im Staatsarchiv Bremen aufbewahrt werden. ${ }^{1}$ Bei den beschriebenen Personen handelt es sich um Menschen aus Afrika bzw. aus Europa, die in unterschiedlicher Weise mit der NMG in Kontakt standen, für sie arbeiteten oder an den von ihr bereitgestellten Angeboten teilnahmen. Im Sommersemester 2016 haben Theologiestudierende an der Carl von Ossietzky Universität Oldenburg kleine Forschungsprojekte durchgeführt, um der kirchengeschichtlichen Bedeutung dieser und weiterer Fotografien nachzugehen. Dafür wurde ein Seminarkonzept mit einer dezidiert postkolonial forschenden Perspektive erprobt, das nicht nur mit den Grundanliegen postkolonialer Ansätze vertraut machen, sondern auch Gelegenheit zur Anwendung bieten sollte. Didaktische ,Erprobungen` von postkolonialen Seminarkonzepten sind deshalb notwendig, weil auch nach Jahrzehnten postkolonialer Forschung und zahlreichen Publikationen die Umsetzung in der kirchengeschichtlichen Lehre zumindest im deutschsprachigen Raum noch ganz am Anfang steht. Ein Blick auf das aktuelle Lehrangebot theologischer Einrichtungen zeigt diese zurückhaltende Aufnahme (post-)kolonialer Themen und Perspektiven. Zudem werden postkoloniale Anfragen zu kulturell bedingten Differenz- und Machtverhältnissen vor allem in spezialisierten Fächern wie der Missionsgeschichte und Ökumene, der Feministischen Theologie oder der Interkulturellen Theologie verhandelt, wodurch der trügerische Eindruck entsteht, als wäre das vermeintliche ,Kerngeschehen' der Theologie von diesen Themen nicht betroffen. Um das zögerliche Interesse der Theologie am Postkolonialismus zu beschreiben, benutzt Michael Nausner das Bild vom „Mauerblümchen“, das sich wenig beachtet in Mauerspalten finden lässt. ${ }^{2}$ Diese Metapher allerdings lässt die irritierende, bisweilen als unangenehm und bedrohlich empfundene Dimension postkolonialer Ansätze unterbelichtet. Diese allerdings scheint mir wesentlich zu sein, um die verhaltene Rezeption in der deutschsprachigen Theologie zu verstehen.

Postkoloniale Ansätze, die sich kritisch mit dem Zustand und der Struktur (post-)kolonialer Gesellschaften auseinandersetzen, wurden vornehmlich an etablierten Universitäten entwickelt und hatten von Anfang an ein großes

1 Die Fotos sind im Staatsarchiv Bremen $(=\mathrm{StAB})$ unter folgenden Titeln zu finden: „Missionsschüler in Keta aus der alten Zeit“ ca. 1853-1867 (StAB 7, 1025-0009); „Schwester H. Rohns mit der Mädchenschule“ ca. 1889-1909 (StAB 7, 1025-1051).

2 Michael Nausner, Die langen Schatten der Nofretete, in: Conc(D) 49/2 (2013), 200-209, 201.207. 
Interesse, die bestehenden akademischen Diskurse herauszufordern. Sie weisen nicht nur auf die Existenz dominanter, hegemonialer Diskurse hin, vielmehr sind sie immer auch darum bemüht, liebgewonnene universitäre Praktiken zu irritieren und forschende Individuen zum Überdenken gewohnter Denkmuster anzuregen. Sie fordern dazu auf, vorhandene Konzepte von Kultur, Gesellschaft, Macht und Identität zu dekonstruieren und auf eine Weise neu zu denken, mit der die Dynamik und Vielschichtigkeit des menschlichen Daseins stärker aufgegriffen wird. Diese hinterfragende und bisweilen schmerzende Provokation des Postkolonialismus mag Anteil daran haben, dass viele Forschende mit Desinteresse oder Abwehr reagieren. Auch bei den Vorbereitungen zur Konferenz, die diesem Sammelband vorausging, stieß die Beschäftigung mit den Ansätzen des Postkolonialismus verschiedentlich auf deutliche Ablehnung. ${ }^{3}$

Ein weiterer Grund für die fehlende Rezeption in der Lehre mag in der Schwierigkeit begründet liegen, die Ansätze didaktisch sinnvoll aufzubereiten. Weil viele Schriften des Postkolonialismus eine hohe inhaltliche Komplexität mit einer hohen sprachlichen Sperrigkeit kombinieren, gestaltet sich die didaktische Vermittlung der Ansätze häufig schwierig. Die Herausforderung für die Lehrenden besteht darin, die postkolonialen Impulse für Theologiestudierende nachvollziehbar zu machen und ihre Anregungen auf den zeitlich stark begrenzen Lehr- und Lernalltag eines theologischen Seminars zuzuspitzen.

Dieser Beitrag widmet sich deshalb der Frage: Wie kann eine postkoloniale, machtkritische Analyseperspektive für kirchengeschichtliche Seminare fruchtbar gemacht werden? Dafür wird ein bereits durchgeführtes Seminar dargestellt, das Missionsgeschichte, die damit verwobene Kolonialgeschichte und ihre bis heute andauernden Folgen für den Forschungsalltag in den Blick rückt. Methodisch war das Seminar an dem Konzept des forschenden bzw. forschungsbasierten Lernens orientiert.

Die Kombination von forschendem Lernen und postkolonialen Perspektiven hat sich als besonders hilfreich und produktiv erwiesen. Auf

3 Die anhaltende Abqualifizierung machtkritischer Analysen und ihre Herabsetzung als "Randthema“ der Theologie belegt Judith Gruber anhand von exemplarischen Gesprächen und Email-Korrespondenzen aus dem akademischen Kontext. Ihre Beispiele, die sich meiner Erfahrung nach schnell zu einer großen Sammlung ergänzen ließen, werfen dringliche Fragen auf: Worin bestehen die vermeintlichen „Kernthemen“ deutschsprachiger Theologie und welche Bedeutung kommen dabei den Kategorien Gerechtigkeit, Befreiung und Solidarität zu? Vgl. Judith Gruber, Wider die Entinnerung. Zur postkolonialen Kritik hegemonialer Wissenspolitiken in der Theologie, in: ANDREAS NeHRING/Simon WieSGiCKL (Hg.), Postkoloniale Theologien II. Perspektiven aus dem deutschsprachigen Raum, Stuttgart 2018, 23-37, 23-24. 
hochschuldidaktischer Ebene ist eine positive Wechselwirkung beider Ansätze festzustellen: Einerseits erfahren forschungsbasierte kirchengeschichtliche Lehrkonzepte durch postkoloniale Fragestellungen eine machtkritische Vertiefung. Andererseits stellen forschungsbasierte Seminare einen geeigneten Lehr- und Lernkontext bereit, um postkoloniale Überlegungen für Studierende sinnvoll zu konkretisieren. Diese Wechselwirkung soll im Folgenden näher beleuchtet werden.

\section{$2 \quad$ Postkolonialismus und Hochschuldidaktik}

Postkolonial arbeitende Menschen weisen seit über dreißig Jahren darauf hin, dass die Beschäftigung mit dem Kolonialismus und seinen unbearbeiteten Folgen eine bleibende Aufgabe von akademischer Forschung und Lehre ist. Im Rahmen postkolonialer Ansätze finden sich zahlreiche Überlegungen, die zum Nachdenken über Lernprozesse im Allgemeinen und über Hochschuldidaktik im Speziellen einladen. Die postkoloniale Denkerin Gayatri Chakravorty Spivak beispielsweise hat sich in zahlreichen Schriften und Interviews zum Lernen und Lehren, zu Bildung und Unterricht geäußert.4 Ihre eigenen Erfahrungen in verschiedenen Bildungskontexten, als Professorin an der Columbia-Universität und in Sommermonaten als Organisatorin von Weiterbildungsprogrammen in ländlichen Gebieten Westbengalens, sind dabei prägend gewesen. ${ }^{5}$ Ihre Lehrtätigkeiten in subalternen und privilegierten Bildungsräumen hängen für Spivak eng zusammen und beeinflussen ihre Überlegungen zur Bildung gleichermaßen. ${ }^{6}$ So stehen im Zentrum ihres Ansatzes der Zusammenhang von Wissen und Macht sowie die Ambivalenzen von Bildungsprozessen. Um

4 Pädagogische Fragen werden u.a. verhandelt in: Gayatri Chakravorty Spivak, An Aesthetic Education in the Era of Globalization, Cambridge (MA) 2012; DIEs., Outside in the Teaching Machine, London/New York 1993; DIES., „Why Study the Past?“, in: MLQ 73.1 (2012), $1-12$.

5 Vgl. Stephen Morton, Gayatri Spivak. Ethics, Subalternity and the Critique of Postcolonial Reason, Key Contemporary Thinkers, Cambridge 2007, 161. Die 1997 gegründete und nach ihren Eltern benannte Stiftung ,Pares Chandra and Sivani Chakravorty Memorial rural Education Project' finanziert Bildungseinrichtungen im nordindischen Westbengalen.

6 Sie selbst hebt die Parallelität dieser beiden Lehrtätigkeiten in Indien und den USA hervor: „I quite resolutely do not make a distinction between the two kinds of teaching; my goals in the two kinds of teaching are the same - to develop the intuitions of democracy." Dieses Zitat und weitere Ausführungen zu Spivaks pädagogischer Intention sind nachzulesen in dem Interview: Occupy Education. An Interview with Gayatri Chakravorty Spivak by Rahul K. Gairola, https://politicsandculture.org/2012/og/25/ occupy-education-an-interview-withgayatri-chakravorty-spivak/ [Zugriff: 30.9.2017]. 
anzuzeigen, dass Denken, Sprechen und Forschen in gewisser Weise immer ein gewalttätiger Akt ist, verwendet sie den Begriff der „epistemischen Gewalt" (epistemic violence). Koloniale Gewalt hat immer eine epistemische Dimension, insofern die Kolonialmächte die Produktion und Vermittlung von Wissen mitbestimmen und für ihre Zwecke nutzen, indem sie beispielsweise die Gruppe der „Anderen“ diskursiv hervorbringen, gewissermaßen erst als solche erfinden. ${ }^{7}$

Epistemische Gewalt äußert sich zudem darin, dass sich in hegemonialen Diskursen entscheidet, wem zugehört wird, wer sprechen kann, wer als wissenschaftlich gilt und wer vom öffentlichen Diskurs ausgeschlossen bzw. von ihm vereinnahmt wird. Bildungseinrichtungen als Ort der Befreiung und Befähigung zu idealisieren, wäre deshalb zu simplifizierend. In diesem Sinne sind auch kirchengeschichtliche Seminare vor die Herausforderung gestellt, sich selbstkritisch mit den Fragen nach dem Verhältnis von Wissen, Macht und Repräsentation auseinanderzusetzen. Um solche machtkritischen Perspektiven in der kirchengeschichtlichen Lehre zu stärken, bietet sich das didaktische Konzept des forschenden Lernens an.

\section{$3 \quad$ Was ist Forschendes Lernen?}

Im Nachdenken über Möglichkeiten eines Lernkulturwandels in der universitären Lehre sind in den letzten Jahren didaktische Konzepte, die das Lernen im Forschen verankern, wieder neu in den Fokus gerückt. Als 2001 der Wissenschaftsrat in seiner Empfehlung zur zukünftigen Struktur der Lehrerausbildung formulierte, die „Hochschulausbildung soll die Haltung forschenden Lernens einüben und fördern“8, griff man damit ein Anliegen auf, das 30 Jahre zuvor bereits von der Bundesassistentenkonferenz ausführlich dargestellt worden war. ${ }^{9}$ Im Sinne der Humboldt'schen Universitätskonzeption sollte demnach die Verbindung von Forschung und Lehre als ein konstitutives

7 „Das klarste Beispiel für eine solche epistemische Gewalt ist das aus der Distanz orchestrierte, weitläufige und heterogene Projekt, das koloniale Subjekt als Anderes zu konstituieren. Dieses Projekt bedeutet auch die asymmetrische Auslöschung der Spuren dieses Anderen in seiner prekären Subjektivität bzw. Unterworfenheit." Gayatri Chakravorty SPIVAK, Can the Subaltern Speak? Postkolonialität und subalterne Artikulation. Übers. AleXANDE R Joskowicz, Stefan Nowotny, Einl. Hito Steyerl, Wien 2008 (erstmals 1988 erschienen), 42.

8 Wissenschaftsrat, Empfehlungen zur künftigen Struktur der Lehrerausbildung, Berlin 2001, 41.

9 Bundesassistentenkonferenz (BAK), Forschendes Lernen - Wissenschaftliches Prüfen, Bonn 1970 (Neudruck Bielefeld 2009). 
Merkmal universitärer Ausbildung ernstgenommen und Forschendes Lernen als ein Kernelement der Hochschuldidaktik gefördert werden.

Auch die Theologie steht als geisteswissenschaftliches Fach in Tradition einer engen Verbindung von Lernen und Forschen. So hat neben Alexander von Humboldt auch der Theologe Friedrich Daniel Ernst Schleiermacher (17681834) die forschende Grundhaltung als Qualitätsmerkmal für gelingendes Studieren hervorgehoben. Schleiermacher sah in Abgrenzung zur schulischen Ausbildung das Besondere der Universität in der "Einleitung eines Prozesses“. Dieser Prozess ziele darauf, „in jedem Denken sich der Grundgesetze der Wissenschaft bewußt zu werden, und eben dadurch das Vermögen, selbst zu forschen, zu erfinden und darzustellen, allmählich in sich heraus[zu]arbeiten“. Den Anstoß dieser Bewegung hin zum Forschen bezeichnete Schleiermacher als das grundlegende "Geschäft der Universität ${ }^{{ }^{110}}$. Wer studiert, so führte er aus, dürfe sich demnach nicht mit dem Memorieren und Rezipieren von Forschungsergebnissen zufriedengeben und müsse von Anfang an dazu ermutigt werden, über die präsentierten Inhalte kritisch forschend nachzudenken. Das Konzept "forschungsbasiertes Lehren im Fokus“ (FliF), wie es an der Carl von Ossietzky Universität Oldenburg praktiziert wird, ähnelt diesen Gedanken Schleiermachers. Auch hier wird das Lernen im Forschen verortet. Der Bielefelder Erziehungswissenschaftler Ludwig Huber hat wegweisende Beiträge darüber verfasst, wie sich dies im konkreten Lehralltag fördern lässt." Studierende sollen selber als Forschende tätig werden, indem sie in kleineren Projekten selbstgewählte Forschungsfragen ergebnisoffen bearbeiten und dabei einen möglichst vollständigen Forschungsprozess durchlaufen. Sie können auf diese Weise im geschützten Rahmen des Seminars Forschungserfahrungen sammeln und zu eigenverantwortlich Lernenden werden. Als Konsequenz daraus werden Seminare als gemeinsame Lernereignisse von Dozierenden und Studierenden verstanden, bei dem die Lehrenden vor allem beratende und unterstützende Rollen innehaben.

10 Friedrich Daniel Ernst Schleiermacher, Gelegentliche Gedanken über Universitäten im deutschen Sinn (1808), in: DERS., Texte zur Pädagogik, Bd. 1, hg.v. Michael Winkle R, Frankfurt am Main 2000, 101-165, 116.

11 Siehe Ludwig Huber, Warum Forschendes Lernen nötig und möglich ist, in: Ludwig Huber/Julia Hellmer/Friederike Schneider (Hg.), Forschendes Lernen im Studium. Aktuelle Konzepte und Erfahrungen, Bielefeld 2009, 9-36. Die Unterscheidung zwischen "forschendem“ und „forschungsbasiertem“ Lernen, die Huber vornimmt, wird im Oldenburger Konzept nicht mitvollzogen. Auch in dem vorliegenden Aufsatz werden beide Begriffe äquivalent und in dem Sinne verwendet, dass Studierende selbst forschend tätig werden. 
In kirchengeschichtlichen Seminaren kann das Konzept beispielsweise umgesetzt werden, indem Studierende eigene Forschungsvorhaben zu Quellen aus der Christentumsgeschichte konzipieren, in Begleitung durchführen und daraus hervorgehende Ergebnisse vor Dritten im Format eines wissenschaftlichen Vortrags präsentieren. Es hat sich bewährt, den Forschungsprozess dafür in kleine Schritte aufzuteilen und diese im Semesterverlauf sukzessive zu erarbeiten, einzuüben und zu reflektieren. $\mathrm{Zu}$ den zentralen Schritten eines kirchengeschichtlichen Forschungsprozesses zählen u.a. folgende Elemente, deren Reihenfolge je nach Fall abgeändert werden kann: das Abstecken eines Forschungsbereichs, die Reflexion von eigenem Vorwissen, Überlegungen zur Relevanz, die Wahl eines konkreten Forschungsgegenstandes, die Entwicklung einer Fragestellung, die Einordnung in den Stand der Forschung, die Formulierung einer These, eine historisch-kritische Quellenanalyse, die Präsentation und Diskussion von (Zwischen-)Ergebnissen sowie die abschließende schriftliche Darstellung der Forschungsergebnisse. ${ }^{12}$ Studierende sollen mit dieser aktivierenden Methode schrittweise lernen, unbekannte Quellen zu erschließen, selbstständig Wissen zu relevanten Themen der Christentumsgeschichte zu erarbeiten, kritisch zu reflektieren und darüber gesprächsfähig zu werden. ${ }^{13}$

Postkoloniale Ansätze sind für dieses Konzept insofern besonders gewinnbringend, dass sie jenes im Forschenden Lernen bereits angelegte (selbst)kritische Moment stärken und vertiefen. ${ }^{14}$ Sie regen dazu an, den Forschungsstand, das Forschungsumfeld sowie das eigene Handeln im Forschungsprozess machtkritisch zu beleuchten. Welche postkolonialen Impulse für das Forschende Lernen relevant sind und wie sich eine postkoloniale Sensibilisierung im Rahmen dieses Ansatzes stärken lässt, soll im Folgenden anhand einzelner Seminarelemente erörtert werden. Zunächst wird dafür kurz das Seminar skizziert.

12 Die einzelnen Elemente bauen zwar grundsätzlich aufeinander auf, überlappen einander aber zugleich an vielen Stellen und können auch zyklisch sein.

13 „Auf aktiven Aneignungsprozessen beruhendes Wissen wird tiefer verarbeitet, besser behalten und bekommt eine größere persönliche Bedeutung als Wissensbestände, die man passiv-rezeptiv aufnimmt. Die beim Forschenden Lernen geforderte Selbständigkeit und Aktivität geht zudem in der Regel mit einer intrinsischen Lernmotivation einher und kann [...] eine dauerhafte Lernbereitschaft begünstigen." WolfGang Fichten, Forschendes Lernen in der Lehrerbildung, in: UlRike Eberhardt (Hg.), Neue Impulse in der Hochschuldidaktik, Wiesbaden 2010, 3-4.

14 Fichten konstatiert, dass Forschendes Lernen eine „fragend-entwickelnde und kritischreflexive Haltung “ fördere, vgl. FiC HTEN, Forschendes Lernen, 4. 
Innerhalb eines Semesters haben ca. 30 Studierende aus zwei Seminaren einen kirchengeschichtlichen Forschungszyklus im Miniformat durchlaufen und postkolonial reflektiert. Das Forschungsfeld und die Methodik waren dem Seminar aus pragmatischen Gründen vorgegeben. Als Grundlage der studentischen Forschung diente die Fotosammlung der NMG. ${ }^{15}$ Diese umfasst insgesamt 5.316 einzeln verzeichnete Schwarz-Weiß-Bilder, die 1846-1965 aufgenommen wurden und heute zur weltweiten Benutzung digital zugänglich sind. ${ }^{16}$ Die teils in Missionszeitschriften abgedruckten, teils unveröffentlichten Fotos stellen in vielfacher Hinsicht eine kirchengeschichtlich und gesellschaftspolitisch relevante Quellensammlung dar. Die Vielfalt von Bildmotiven und Bildkompositionen zeugt von der wechselhaften Geschichte der NMG im Gebiet des heutigen Togo und Ghana, vor allem aber verdeutlicht sie die Bandbreite visueller Repräsentationen des außereuropäischen Christentums im 19. und 20. Jahrhundert. Da zu dieser historischen Fotosammlung noch keine detaillierten Analysen mit postkolonialer Perspektive vorliegen, standen die Dozentin und die Studierenden ganz im Sinne des Forschenden Lernens gemeinsam vor der Herausforderung, ein angemessenes Vorgehen zu entwickeln und Thesen zu entfalten. ${ }^{17}$

Die erste Annäherung an das Forschungsthema erfolgte über eine kritische Lektüre von Missionsgeschichtsdarstelllungen in Lehrbüchern. Dies und die anschließende Reflexion über die eigene Rolle als Forschende wurden zum Anlass genommen, um erste postkoloniale Grundideen zu thematisieren. Daran anschließend wählten alle Teilnehmenden ein Foto aus dem Archiv als Forschungsprojekt aus, wobei es allen vorbehalten blieb, diese Entscheidung noch einmal abzuändern. Gemeinsam arbeiteten sich die Studierenden dann in die Entstehungsgeschichte und das Selbstverständnis der NMG ein. Zudem wurden die einzelnen Arbeitsschritte einer Fotoanalyse anhand von Fallbeispielen erprobt und postkolonial reflektiert, bevor sich die Studierenden

\footnotetext{
15 Zur inhaltlichen Einführung in den Bildbestand eignet sich KonRAd ElmshäUSER, Menschen in Afrika - aus dem Bildarchiv der Norddeutschen Mission, in: BrJ 85 (2006), $8-13$.

16 Die Beständepräsentation des Staatsarchivs Bremen ist zugänglich unter: www. staatsarchiv-bremen.findbuch.net [letzter Zugriff 20.8.2017].

17 Rainer Alsheimer hat eine Monografie zu der Fotosammlung vorgelegt, ohne jedoch die Bildkompositionen, Motive etc. ausreichend kritisch zu beleuchten. Vielmehr verwendet er die Fotografien hauptsächlich in illustrierender Absicht. Vgl. Rainer Alsheimer, Bilder erzählen Geschichten. Eine Fotoanthropologie der Norddeutschen Mission in Westafrika, Bremen 2010.
} 
im weiteren Verlauf des Seminars auf das von ihnen ausgewählte Foto fokussierten. Diese individuelle Forschungsphase wurde durch einen gemeinsamen Archivbesuch im Staatsarchiv Bremen ergänzt, bei dem die Verwendung der jeweiligen Fotografien in Missionszeitschriften untersucht wurde. Insgesamt wechselten im Semesterverlauf Gruppenarbeit, Einzelarbeit, kollegiale Beratung und individuelle Beratungsgespräche mit der Dozentin ab. Auf diese Weise konnten die Studierenden Erfahrungen sowohl mit der individuellen als auch mit der kollaborativen Dimension von Forschung sammeln. Ziel der studentischen Forschungsprojekte war es, eine Fotoanalyse zu erarbeiten und am Ende des Semesters in Form eines Kurzvortrages auf der wissenschaftlichen Konferenz, die diesem Sammelband zugrunde liegt, vor einem international renommierten Fachpublikum zu präsentieren und zu diskutieren. ${ }^{18}$ Wie bei der Abfassung wissenschaftlicher Beiträge üblich konnten die Studierenden die auf der Konferenz erhaltenen Rückmeldungen in ihre Texte einarbeiten, bevor sie die fertige Fassung in schriftlicher Form als Prüfungsleistung bei der Dozentin einreichten..$^{19}$ In welcher Weise postkoloniale Perspektiven und Forschendes Lernen wechselseitig voneinander profitieren, soll anhand von vier Themenfeldern aufgezeigt werden: 1 . Vorwissen und Selbstreflexion, 2. Relevanz und Geschichtsnarrative, 3. Quellenauswahl und Kanonkritik, 4. Quellenanalyse und Irritationen.

\subsection{Vorwissen - Selbstreflexion der Forschenden}

Eine wesentliche Aufgabe forschungsbasierter Seminare besteht darin, Studierenden bei der Entwicklung eines Bewusstseins als Forschende zu unterstützen. Dazu gehören sowohl die Wahrnehmung des eigenen Vorwissens als auch die kritische Reflexion der persönlichen Kontextgebundenheit.

Um das Vorwissen der Studierenden zu aktivieren, begann das Seminar mit der Aufgabe, in kleinen Gruppen eine Idee für ein Cover einer Missionszeitschrift zu entwickeln und diese in einem Foto festzuhalten. Bei der gemeinsamen Analyse der Ergebnisse waren die wiederkehrenden Motive offensichtlich: Häufig wurde die Mission als Begegnung einer Person mit einer Gruppe von Menschen dargestellt. Die Missionierenden wurden bei der Anordnung erhöht positioniert, beispielsweise stehend. Sie hatten oft als einzige ein Buch in der Hand, dessen Inhalt sie den Anwesenden erklärten,

18 Im Tagungsprogramm wechselten sich Blöcke aus Vorträgen von eingeladenen Gästen mit Studierendenvorträgen ab. Diese Mischung hat sich als ertragreich erwiesen. Auf diese Weise konnten Forschende auf ganz verschiedenen Ebenen der wissenschaftlichen Laufbahn miteinander ins Gespräch kommen.

19 Je nach Umfang der zu erbringenden Seminarleistung wurde entweder das Vortragsmanuskript oder eine Weiterentwicklung des Manuskripts eingereicht. 
oder sie hielten Ansprachen an ihre Zuhörerschaft. Dadurch waren sie von der Zielgruppe missionarischer Ansprachen abgegrenzt. Diese wurden zumeist schweigend und mit empfangenden Haltungen dargestellt.

Im anschließenden Gespräch wurden verschiedene studentische Statements zur Mission gesammelt und wiederkehrende Bewertungsmuster identifiziert. Die von Europa ausgehende Mission des 19. Jahrhunderts wurde aufgrund ihrer problematischen Verstrickung in die Kolonialpolitik häufig vor allem mit Zwang, Gewalt und Zerstörung assoziiert und dementsprechend negativ bewertet. Neben der abwertenden Verurteilung gab es aber auch das Phänomen nostalgischer Romantisierung, bei dem missionarische Aktivitäten einseitig mit positiven Errungenschaften wie der Übersetzung der Bibel oder der Einrichtung von Schulen verbunden wird. Als gemeinsames Moment sowohl der idealisierenden als auch der ablehnenden Haltung gegenüber der Mission wurde die vereinfachende Zuschreibung von aktiven und passiven Rollen identifiziert. Wie bereits bei den erstellten Fotografien scheinen einzig die europäischen Missionare eine aktive Rolle innezuhaben und über den Verlauf der Ereignisse zu entscheiden, wohingegen die Missionierten eine passive Rolle zugeschrieben bekamen, in der sie je nach Deutung die Erleidenden oder Empfangenden waren.

Anhand dieser Beobachtungen wurden im postkolonialen Sinne die grundlegenden Zusammenhänge von Dichotomisierung, Essentialisierung, Hierarchisierung und ,Othering thematisiert. Um wirkmächtige Formen binären Denkens aufzuzeigen, ist Edward Saids eindrucksvolles Werk "Orientalismus“ hilfreich. ${ }^{20}$ Said identifiziert darin den ,Orient" als eine im westlichen Diskurs erst geschaffene Kategorie, die ganz verschiedene Gruppen zusammenfasste und je nach Epoche auf ganz verschiedene geographische Regionen bezogen sein konnte. Saids Analyse verdeutlicht, auf welche Weise Zuschreibungen zum Orient während und nach der Kolonialzeit mit Strategien der Macht verbunden waren bzw. sind. Dichotome Zuschreibungen dienten den Kolonialmächten dazu, im Diskurs ein koloniales Gegenüber zu konstruieren und so hierarchische Ordnungen zu stabilisieren. Der "Orient" wurde dafür im vermeintlichen Kontrast zum sog. „Westen“ imaginiert. So galt der "Orient" als entwicklungsbedürftig statt fortschrittlich, wild statt geordnet, faul statt fleißig, hilfebedürftig statt kompetent, impulsiv statt rational, passiv statt aktiv, usw. Indem Kolonialmächte dem Orient defizitäre Eigenschaften zuschrieben und dabei jede Form innerer Differenzen oder Brüche nivellierten, legitimierten sie die hegemonialen Strukturen der von ihnen geschaffenen

20 Edward SAID, Orientalism, New York 1978, in deutscher Übersetzung: Ders., Orientalismus, Frankfurt am Main 2009. 
kolonialen Weltordnung. Said zeigt auf, dass essentialistische und binäre Zuschreibungen fest in den Diskurs von kolonisierenden ebenso wie kolonisierten Gesellschaften eingeschrieben sind. Bis heute prägen sie das Denken und Sprechen über , andere Kulturen“ sowie die Repräsentation „Fremder" in Texten und Bildern. Es gehört zu den unbearbeiteten Folgen des Kolonialismus, dass jene kolonialen Denkmuster, die als Rechtfertigungsstrategien für europäische Kolonialmächte genutzt wurden, bis heute in Belletristik, Presse, Comics, aber auch in wissenschaftlichen Kontexten hartnäckig (re-)produziert werden. Im Seminar besteht bereits bei der Aktivierung von Vorwissen Gelegenheit und zugleich Notwendigkeit, (un)bewusst übernommene Zuschreibungen zu identifizieren und die eigene Verstrickung in die dominanten Diskurse kritisch zu reflektieren.

Neben der kritischen Selbstreflexion eigener Vorannahmen und Erwartungshaltungen ist auch die Rolle als Forschende(r) im Forschungsprozess zu bedenken. Eindringlich weist Spivak forschend arbeitende Menschen darauf hin, die Situiertheit des eigenen Wissens nicht aus den Augen zu verlieren. Man dürfe die eigene Geschichte als westliche Intellektuelle und die damit verbundenen Privilegien der Macht im Prozess der Interpretation nicht ignorieren. ${ }^{21}$ In Seminaren kann dafür die Praxis der akademischen Selbstdistanzierung eingeübt werden. Zum Vorbild kann hier jene Praxis auf internationalen Konferenzen dienen, bei der Tagungsbeiträge mit einer kurzen Auskunft über den eigenen sozio-kulturellen Hintergrund oder mit biographischen Bezügen zum Forschungsthema begonnen werden. Ziel ist die selbstreflexive Wahrnehmung hermeneutisch relevanter Voraussetzungen wie Hautfarbe, Bildungsstand, persönliche Erfahrungen mit dem Forschungsthema. Eine solche Form der Selbstdistanzierung trägt dazu bei, ein Bewusstsein für die Kontextgebundenheit der eigenen Forschungsposition, aber auch für die eigenen Verstrickungen in den westlichen, kirchlichen und universitären Diskursen zu entwickeln.

Für Spivak ist Lernen kein lineares Geschehen, bei dem Wissen angehäuft wird. Lernen ist für sie ein dialektischer Prozess von Lernen und Verlernen, bei dem bereits vorhandenes Wissen kritisiert und manchmal sogar verlernt werden muss. Verlernt werden muss beispielsweise, die eigenen Privilegien und den Status als Vorteil wahrzunehmen, weil sie zu einem Problem werden, wenn es um die Erforschung von marginalisierten Gruppen geht. ${ }^{22}$

\footnotetext{
21 SPIVAK, Can the Subaltern Speak?, 28.39.

22 "My project is the careful project of un-learning our privilege as our loss“, GAYATRI Chakravorty Spivak, The Post-Colonial Critic. Interviews, Strategies, Dialogues, hg.v. SARAH HARASYM, New York/London 1990, 9.
} 


\subsection{Relevanz des Themas - Dekonstruktion von Geschichtsnarrativen}

In einem weiteren Schritt wurden im Seminar ausgewählte kirchengeschichtliche Überblicksdarstellungen und Lehrbücher auf die Frage hin untersucht, welcheBedeutungsiedemeuropäischen Kolonialismus, derMissionsgeschichte und außereuropäischen Formen des Christentums einräumen und wie die entsprechenden Darstellungen strukturiert sind. Gerade in Lehrbüchern lassen sich unverkennbare Nachwirkungen kolonialer Deutungsmuster aufzeigen, die sich entweder durch Verdrängung oder durch verzerrende Narrative bemerkbar machen. Zwei Beispiele seien hier kurz angedeutet:

Das kirchengeschichtliche Repetitorium von Sommer/Klahr, das inzwischen in fünfter Auflage erschienen ist, listet die Namen und Gründungsjahre zahlreicher Missionsgesellschaften, ohne weiter auf Zusammenhänge und Bedeutung einzugehen..$^{23}$ Das Stichwort Kolonialismus wurde erst gar nicht in das Register aufgenommen. Auch in dem Lehrbuch der Kirchen- und Dogmengeschichte von Wolf-Dieter Hauschild sucht man im umfangreichen Sachregister vergeblich nach diesem Begriff. Spivak hat darauf hingewiesen, dass gerade die Ignoranz bestimmter Zeugnisse und Zusammenhänge ein unbewusster Akt der Machterhaltung und Abschottung gegenüber nichtwestlichen Ansprüchen darstellen kann. ${ }^{24}$

Die ökumenische Sammlung „Kirchengeschichtliche Grundthemen“, die sich dezidiert an angehende Lehrer und Lehrerinnen richtet, konstatiert zunächst eine nicht näher definierte „Brisanz des Themas,Weltmission““25, und versucht dann diese anhand exemplarischer Einblicke in die Geschichte der Mission nachzuweisen. Um die Bandbreite missionarischer Positionierungen herauszustellen, skizzieren die Autoren Fallbeispiele, in denen die Missionare je nach historischer Konstellation und missionarischem Konzept auf unterschiedliche Weise aktiv waren, indem sie Schwächere unterdrückten oder für Unterdrückte eintraten, Zwang ausübten oder für Bildung sorgten, Wehrlose beschützten oder Innovationen förderten. Die europäischen Missionare werden hier als entscheidende Protagonisten des Missionsgeschehens

23 Vgl. Wolfgang Sommer/Detlef Klahr/Marcel Nieden, Kirchengeschichtliches Repetitorium. Zwanzig Grundkapitel der Kirchen-, Dogmen- und Theologiegeschichte, Göttingen u.a. ${ }^{52012,} 223$.

24 Sie benutzt die Formulierung „sanctioned ignorance“. GAYATri Chakravorty SPIVAK, A Critique of Post-Colonial Reason. Toward a History of the Vanishing Present, Cambridge (MA)/London 1999, 2.164 u.ö.

25 Herbert Gutschera/Jörg Thierfelder, Weltmission in der Neuzeit, in: Jörg Thierfelder/Herbert Gutschera/Rainer Lachmann (Hg.), Kirchengeschichtliche Grundthemen. Historisch - systematisch - didaktisch, ThLL 3, Göttingen ${ }^{4}$ 2014, 252- 270; hier: 253. 
stilisiert; sie evozierten so je nach Haltung gegenüber ihrer Zielgruppe ein positives oder negatives Bild von Mission. Die wichtige Gruppe der nichteuropäischen Mitarbeitenden bleibt unterbeleuchtet, ebenso die Vielschichtigkeit und Ambivalenz von Machtverhältnissen sowie deren Rückwirkungen auf europäische Gesellschaften.

Vor diesem Hintergrund wurde im Seminar erörtert, warum aus kirchengeschichtlicher Sicht eine Auseinandersetzung mit der Epoche des Kolonialismus und mit der Missionsbewegung im 19. Jahrhundert sinnvoll ist. Dafür ergab sich eine doppelte Argumentationsweise: Zum einen gehört es zu den Aufgaben der kirchengeschichtlichen Disziplin, historische Machtkonstellationen kritisch zu reflektieren, an denen kirchliche Institutionen und Akteure mit einem christlichen Selbstverständnis beteiligt waren. Die Christentumsgeschichte kolonisierter und kolonisierender Gesellschaften mit ihren jeweiligen Bezügen zueinander, das Verhältnis von Mission und Kolonialpolitik, aber auch die theologischen Rechtfertigungsmuster von kolonialer Herrschaft und imperialem Machtanspruch sind Teil der europäischen Vergangenheit, die nicht einfach ausgeblendet werden dürfen.

Zum anderen ist die Beschäftigung mit kolonialen Praktiken sowie mit (theologischen) Begründungsmustern kolonialer Macht unverzichtbar, um ihr Weiter- und Nachwirken in der neueren Christentumsgeschichte des 20. und 21. Jahrhunderts wahrnehmen sowie hinterfragen zu können. Der Zusammenhang von Theologie und kolonialen Denkmustern ist dabei nicht nur eine Angelegenheit der Vergangenheit, sondern auch eine der Gegenwart. Eine umfassende postkoloniale Revision kirchengeschichtlicher Lehrbücher steht noch aus. So sind die akademischen Diskurspraktiken des Faches nicht hinreichend entkolonialisiert, solange das von den Kolonialmächten etablierte dichotome Repräsentationssystem reproduziert wird, welches die Missionierten ohnmächtig und die Missionare machtvoll imaginierte.

Als wichtige Bezugstexte sind an dieser Stelle die Arbeiten von Mary Louise Pratt und Homi K. Bhabha zu nennen. Pratt beschreibt die Interaktion von Menschengruppen verschiedener kultureller Prägungen, die vorher räumlich getrennt lebten, als eine Contact Zone. In diesen Kontaktzonen werden trotz der Abhängigkeitsverhältnisse auf dynamische Weise Handlungsmöglichkeiten verhandelt, neue Sprachformen ausgebildet und neue Kommunikationswege erprobt. Zuschreibungen von rein passiven oder rein aktiven Rollen in der kulturellen Begegnung sind somit obsolet. ${ }^{26}$

26 Um das Konzept der Contact Zone zu verdeutlichen, verweist sie auf den Unterrichtsraum: „All the students in the class had the experience [...] of having their cultures discussed and objectified in ways that horrified them; all the students experienced face-to-face the 
Bhabha arbeitet auf eindrückliche Weise heraus, dass Kulturen, Gesellschaften und Identitäten niemals in sich abgeschlossene, stabile Entitäten sind, sondern im Gegenteil immer hybride, fluide und miteinander verflochtene Größen darstellen. Das von ihm etablierte Konzept des Third Space hebt die Dynamik und Komplexität hervor, die die Produktion von kultureller Differenz auszeichnet. ${ }^{27}$ Die Konzepte des Third Space und der Contact Zone betonen die gegenseitige Beeinflussung innerhalb der Begegnungsräume, die keinen der Beteiligten unverändert lässt. Das Missionsgeschehen kann somit nicht mehr nur als einseitige Einflussnahme im Sinne eines SenderEmpfänger-Modells interpretiert werden, sondern muss als die gemeinsame Geschichte von Missionierten, Missionsverweigerern und Missionierenden gesehen werden. In den Blick rücken dabei die vielfältigen Verflechtungen, wechselseitige Beeinflussungen, kulturelle Zwischenräume, transformierende Beziehungen und das Neue, das entsteht. ${ }^{28}$

Die Aufgabe von postkolonial sensiblen Forschungsprojekten besteht darin, die aktuelle Forschungslage und ihre Vermittlung in Lehrbüchern nicht nur wahrzunehmen, sondern auch kritisch zu reflektieren. Dazu gehört die Irritation und Dekonstruktion der angebotenen Narrative.

\subsection{Quellenauswahl-Kanonkritik und Kanonerweiterung}

Zum Forschungsprozess gehört für die Studierenden im Seminar auch die Auswahl des Forschungsgegenstandes. Auch dieser Vorgang kann mit Studierenden postkolonial beleuchtet werden. So ist beispielsweise wahrzunehmen, dass die in der Kirchengeschichte als ,zentral' angesehenen Quellen häufig dem europäischen Kontext entstammen. Zeugnisse von afrikanischen oder asiatischen Varianten des Christentums finden kaum Beachtung. Der bengalische Historiker Dipesh Chakrabarty hat im Kontext seiner berühmten Forderung, Europa in der Geschichtsschreibung zu „provinzialisieren“, auf die Tatsache hingewiesen, dass im Bereich der akademischen Forschung eine „asymmetrische Unkenntnis“ zu erkennen ist. Das Phänomen zeige sich darin, dass Forschende außerhalb Europas die westliche Geschichte grundsätzlich

ignorance and incomprehension, and occasionally the hostility of others [...] Along with rage, incomprehension, and pain, there were exhilarating moments of wonder and revelation, mutual understanding, and new wisdom - the joys of the contact zone." MARY Louise Pratt, Arts of the Contact Zone, in: Profession 91 (1991), 33-40, 39. Siehe auch Judith Becker (Hg.), European Missions in Contact Zones. Transformation through Interaction in a (Post-)Colonial World, VIEG.B 107, Göttingen 2015.

27 Номі К. Внавна, The location of culture, London/New York 1994; dt. Ausgabe: Die Verortung der Kultur, Tübingen 2000.

28 Vgl. Katja Heidemanns, Art. Mission/Missionarin (kath.), in: WFT 2002, 411-413. 
mit einbeziehen, wohingegen europäische Forschende die nicht-westliche Geschichte kaum oder gar nicht beachten. Eine mentale Dekolonisierung stehe somit noch ganz am Anfang. ${ }^{29}$

Um die eurozentrische Verengung bzw. nationale Selbstbezogenheit der deutschsprachigen Theologie zu unterwandern, ist in einem ersten Schritt die Ausweitung des kirchengeschichtlichen Quellenkanons notwendig. ${ }^{30}$ In jüngerer Zeit werden vermehrt Versuche unternommen, die durch hegemoniale Diskurse verdrängten und vergessenen Geschichten sowie biographischen Schicksale marginalisierter Menschengruppen ins Bewusstsein zu rücken. Stärker als bisher wird dafür auch auf Materialien aus nicht-europäischen Handlungsräumen bzw. Kontaktsituationen zurückgegriffen, und es werden polyzentrische Ansätze verfolgt. ${ }^{31}$

Die Erweiterung des Quellenkanons sollte auch nicht-textliche Quellen einbeziehen, da eine einseitige Sprachfixierung einen Großteil historischer Zeugnisse und Perspektiven verdrängt. ${ }^{32}$ So waren auch in der Kolonialzeit visuelle Reproduktionen von kolonialen Vorstellungen besonders wirkmächtig, da sie in Schul- und Lehrbüchern, in der Tagespresse, in Kinderbüchern oder wie im Fall der Mission vor allem in Missionszeitschriften und auf Postkarten abgebildet waren. Bereits zum Ende des 19. Jahrhunderts wurden dabei

29 Dipesh Chakrabarty, Europa provinzialisieren. Postkolonialität und die Kritik der Geschichte, in: Sebastian Conrad/Shalini Randeria (Hg.), Jenseits des Eurozentrismus. Postkoloniale Perspektive in den Geschichts- und Kulturwissenschaften, Frankfurt/New York 2002, 283-312, bes. 284.

30 In einem weiteren Schritt wäre dann im Sinne von Chakrabarty nach den Analysekriterien zu fragen, mit denen diese Quellen bearbeitet werden, da auch dort westliche Konzepte vorherrschen.

31 Dies schlägt sich in entsprechenden Veröffentlichungen zur globalen Christentumsgeschichte nieder, über die Frieder Ludwig einen Überblick bietet. Vgl. Frieder Ludwig, Globale Christentumsgeschichte, in: VF 57/2 (2012), 106-117. Wichtige Beiträge finden sich in der von Klaus Koschorke und Johannes Meier herausgegebenen Reihe „Studien zur Außereuropäischen Christentumsgeschichte (Asien, Afrika, Lateinamerika)“, Göttingen 1998ff.; Wiesbaden 2002ff. und in KLAUs Kosc HоRкE (Hg.), Außereuropäische Christentumsgeschichte (Asien, Afrika, Lateinamerika), 1450-1990, Neukirchen-Vluyn ${ }^{4}$ 2012. Auch die neueren Arbeiten von Judith Becker zur Missionsgeschichte stellen einen wichtigen Beitrag dar: Vgl. Judith BeCKer, Conversio im Wandel. Basler Missionare zwischen Europa und Südindien und die Ausbildung einer Kontaktreligiosität, 1834-186o ,VIEG 238, Göttingen 2015.

32 Eine Ergänzung der sprachlichen durch visuelle Quellen, was den verstärkten Bezug auf Fotografien einschließt, ist in der Forschung seit den 199oer Jahren zu beobachten. W.J.T. Mitchell prägte dafür 1992 den Begriff ,pictorial turn', Gottfried Boehm 1994 den Begriff ,iconic turn'. Sie und andere rückten visuelle Spuren und Bildlichkeit zunehmend in das Zentrum des Interesses von Forschung. Vgl. dazu Doris BACHMAnN-Medick, Cultural Turns. Neuorientierungen in den Kulturwissenschaften, Hamburg 22007, 329-380. 
Holz- und Kupferstiche durch das neue Medium der Fotografie abgelöst. ${ }^{33}$ Fotografien waren von Anfang an Ausdrucksform sowohl hegemonialer Macht einer westlich-weißen Kolonialinstanz als auch ihrer Unterwanderung. Sie eignen sich deshalb, um eine postkoloniale Bildkritik einzuüben, die sowohl Hierarchisierungen und Abgrenzungen als auch Verflechtungen und gegenseitige Bezugnahmen in den Blick nimmt.

Eine Ausweitung des kirchengeschichtlichen Kanons kann zudem gefördert werden, indem Lehrveranstaltungen nicht nur auf Quelleneditionen, sondern vermehrt auf Archive zugreifen würden. Im Seminar wurde deshalb den Studierenden zunächst eine Auswahl von Fotografien aus dem Repertoire der NMG vorgestellt und dann der Online-Zugang zum Fotoarchiv erläutert, damit sie sich selbst einen Überblick über die Fotografien verschaffen können. Ein Besuch im Bremer Stadtarchiv wurde genutzt, um Fragen nach der Verwendung der jeweiligen Fotografien in Missionszeitschriften zu untersuchen. Ein Archivbesuch ermöglicht Theologiestudierenden somit nicht nur Wissen darüber, wie sie später in ihrer Tätigkeit Zugang zu Quellenmaterial bekommen, sondern auch die Erfahrung, welche Bedeutung die Materialität, der Zustand und die Aufbewahrung für die Quellenanalyse haben.

Während des Archivbesuchs bot es sich zudem an, das Archiv selbst als Ort von Wissens- und Machtproduktion mit Studierenden postkolonial zu reflektieren. Als Orte der Wissensspeicherung sind Archive Ergebnisse von konkreten Entscheidungen, was als aufbewahrenswert erachtet wird, wie Fundstücke zusammengeordnet werden, wie etwas gekennzeichnet bzw. betitelt wird. Damit bestimmen Archive die Erinnerungskultur wesentlich mit. ${ }^{34}$

\subsection{Quellenanalyse-Multiperspektivität, Fehler und Irritationen}

Alleine mit der Erschließung neuer Quellen wäre allerdings im Sinne des Postkolonialismus noch nichts gewonnen, wenn nicht auch der Umgang mit der Quelle zum Thema gemacht würde. Sowohl die Fragen, die an das Foto gerichtet werden als auch die methodische Herangehensweise sind dafür bedeutsam. Neuere Veröffentlichungen zum Umgang mit Missionsfotografien bieten hier wichtige Anregungen. ${ }^{35}$

33 Vgl. Andreas EckL, Ora et labora. Katholische Missionsfotografie aus den afrikanischen Kolonien, in: Marianne Bechiaus-Gerst/Sunna Gieseke (Hg.), Koloniale und postkoloniale Konstruktionen von Afrika und Menschen afrikanischer Herkunft in der deutschen Alltagskultur, Frankfurt am Main 2006, 231-250; hier 231-232.

$\mathrm{Zu}$ postkolonialen Anfragen an Archive siehe den Beitrag von Gladson Jathanna in diesem Sammelband.

35 Zu nennen ist hier das von Judith Becker, Katharina Stornig und Christoph Nebgen 2016 in Mainz organisierte internationale Kolloquium, aus dem ein Sammelband mit 110 
Machtkritische Impulse des Postkolonialismus aufnehmend haben sich die Forschungsprojekte den Fragen gewidmet, wie Menschen(gruppen) aus den Missionsgebieten auf Fotografien abgebildet werden. Die ausgewählten Fotografien zeigen Einzelpersonen, Menschengruppen und zwischenmenschliche Interaktion. Abgebildet sind beispielsweise Missionare bei der Predigttätigkeit mit Zuhörenden, Missionare mit ihren Familien und Mitarbeitenden, gemischte Gruppen afrikanischer und europäischer Pastoren, Einzelpersonen auf Reisen und bei der Überquerung von Flüssen oder Brücken, afrikanischen Frauen mit ihren Kindern, Tauf- und Hochzeitsgesellschaften, afrikanische Frauenvereine u.v.m. Viele Studierende mit dem Berufsziel Lehramt wählten Abbildungen zur Thematik von Bildung: Schulklassen unterschiedlichen Alters mit Lehrpersonal oder einzelne Missionsschüler.

Diese Fotografien wurden von den Studierenden im Laufe des Semesters einer umfangreichen Analyse unterzogen, die unter anderem auch eine ikonographische und serielle Untersuchungsebene umfasste. ${ }^{36}$ Im Interesse der Analysen standen der Konstruktionscharakter der Fotografien und die damit einhergehenden visuellen Darstellungsstrategien. ${ }^{37}$ Die fotografischen Zeugnisse aus der Missionsarbeit hatten innerhalb des missionarischen Diskurses vielfältige Funktionen: Sie sollten missionarische Erfolge

Abbildungen hervorgegangen ist. Vgl. Judith Becker /Katharina Stornig (Hg.), Menschen - Bilder - Eine Welt. Ordnungen von Vielfalt in der religiösen Publizistik um 1900, VIEG 118, Göttingen 2018. Eine umfangreiche Analyse missionarischer Fotografien aus dem afrikanischen Kontext bietet: T. JAск Tномpson, Light on Darkness? Missionary Photography of Africa in the Nineteenth and Early Twentieth Century, Grand Rapids (MI)/Cambridge (UK) 2012. Bemerkenswert sind auch die Arbeiten von Katharina Stornig, die am Beispiel katholischer Missionsarbeit viele grundlegende Erkenntnisse zur Missionsfotografie erarbeitet. Vgl. Katharina Stornig, Vielfache Bedeutungen. Missionsfotografie zwischen Neuguinea und Europa, 1899-ca. 1930, in: Österreichische Zeitschrift für Geschichtswissenschaften 24/2 (2013), 114-139.

36 Die historische Fotoanalyse beschäftigt sich unter anderem mit den Fragen: Wo und wann ist die Fotografie entstanden? Wer war an ihrer Produktion beteiligt? Was war der Anlass der Entstehung und wem wurde sie zugänglich gemacht? Wie ist sie heute aufbewahrt? Welche Wirkungsgeschichte hat das Bild? Welche formalen Gestaltungsprinzipien sind zu erkennen? Welche (biblischen) Motive lassen sich identifizieren? Welche Interpretationsmöglichkeiten gibt es? Welche Besonderheiten kennzeichnet das Foto im Vergleich zu ähnlichen Abbildungen? Zur Einführung in die Methodik siehe JENS JÄGER, Fotografie und Geschichte, Frankfurt am Main/New York 2009 und UlRike PILARCZYK / Ulrike Mietzner, Das reflektierte Bild. Die seriell-ikonografische Fotoanalyse in den Erziehungs- und Sozialwissenschaften, Bad Heilbrunn 2005.

37 „Eine Auseinandersetzung mit dem Konstruktionscharakter von Fotografien wirft die Frage auf, was Missionarinnen und Missionare als abbildungswürdig, herzeigbar oder fotografierenswert erachteten und was sie mit ihrer Wahl eines Bildausschnitts ausschlossen.", Stornig, Vielfache Bedeutungen, 118. 
dokumentieren, die Notwendigkeit missionarischen Engagements demonstrieren, die Spendenbereitschaft fördern und für den Missionsdienst mobilisieren, aber auch emotionale und geistliche Verbindungen zwischen Missionsgemeinden und Unterstützerkreisen stärken. ${ }^{38}$ Zugleich waren sie aber auch in einem allgemeineren Sinne Ausdruck des spezifischen Selbstund Weltverständnisses aller derjenigen Menschen, die an ihrer Produktion mitgewirkt haben. Die Studierenden haben untersucht, welche Rolle die Konstruktionen von „Fremdem“ und die Platzierung von „Bekanntem“ in den Bildern hatten, und inwiefern binäre Repräsentationsmodelle aufgebrochen wurden. Das Format verschiedener Fallbeispiele ermöglicht es, den Variantenreichtum visueller Repräsentation sowie die Vielfalt der darin enthaltenen Brüche vorhandener Machtstrukturen aufzuzeigen. ${ }^{39}$ Während der Präsentation der studentischen Forschung auf der Konferenz hat sich herausgestellt, dass die Identifizierung von dichotomen Zuschreibungen in der Fotografie deutlich einfacher ist als die Identifizierung der Brüche und Spannungen. Die Identifizierung und Reflexion von Machtverhältnissen führt in einem ersten Schritt zunächst einmal dazu, dass diese ins Bewusstsein rücken und (re)produziert werden. Um diese binären Strukturen und Festschreibungen von Identitäten nach ihrer Identifizierung in einem zweiten Schritt aufzubrechen, bedarf es einer Sensibilisierung für die kleinen Störungen, Sprünge und Dissonanzen, die einfachen Zuschreibungen entgegenstehen. Ich möchte deshalb mit einigen didaktischen Anmerkungen schließen, die es noch weiter auszuloten und zu ergänzen gilt:

\subsubsection{Einübung von Multiperspektivität}

Die Multidimensionalität einer Fotografie kann herausgearbeitet werden, indem die abgebildete Situation aus verschiedenen historischen Rollen heraus beschrieben wird. Die abgelichtete schwarze Frau, der weiße Missionar neben ihr, der Fotograf hinter der Kamera, der nichtabgebildete Kolonialbeamte, die europäischen Lesezirkel der Missionszeitschrift, die Verwandten im Nachbarort der Mission, sie alle haben eine eigene Sicht auf die Szene. Sich diese Perspektiven zu imaginieren, kann Studierenden dabei helfen, eine Quelle ,gegen den Strich` zu lesen. Ziel ist es, möglichst viele Zugänge,

38 Vgl. David Morgan, The Sacred Gaze. Religious Visual Culture in Theory and Practice, Berkeley/Los Angeles 2005, 147-19o; KATHARINA StorNig, Authentifizierung kultureller Begegnungen durch Fotografie. Über die Verwendung von Fotos als Spuren in der transnationalen Spendenwerbung im 19. Jahrhundert, in: Saec. 66/2 (2016), 207-228.

39 Anregend ist dabei die Arbeit zu verschiedenen visuellen Fallbeispielen von ViкTORIA Schmidt-Linsenhoff, Ästhetik der Differenz. Postkoloniale Perspektiven vom 16. bis 21. Jahrhundert, 2 Bde., Marburg 2010. 
Perspektiven, Fragen und Thesen auszuprobieren und sich erst dann an eine ,schlüssige‘ Interpretation zu wagen.

\subsubsection{Offenheit für Fehler entwickeln}

Spivak hat in ihrem Aufsatz ,Can the Subaltern Speak?' aufgezeigt, wie eng Repräsentation im Sinne eines "Sprechens für jemanden“ mit Instrumentalisierung und Vereinnahmung einhergeht. ${ }^{40}$ Leicht werden Menschen(gruppen), für die gesprochen wird, zur Projektionsfläche für eigene Anliegen. Diese Gefahr sieht sie bei den weißen Kolonialbeamten der Vergangenheit ebenso wie bei westlichen Akademikern der Gegenwart. Die Grenzen des Deutens und Verstehens, die hier angedeutet sind, betreffen auch die Analyse von Fotografien. Denn in einer Fotografie kann man manchmal das sehen, was in Texten nicht thematisiert wurde. Man kann unausgesprochene Ordnungsmuster und ihre unausgesprochene Irritation entdecken. Man sieht die Haltung und Gesten von Menschen, die sich in schriftlicher Form nicht geäußert haben bzw. deren Worte im hegemonialen Diskurs verdrängt worden sind. Das macht die Attraktivität der Quelle aus und begründet zugleich die mit ihr verbundene hermeneutische Herausforderung. Deshalb kann bei Interpretationen nicht Fehlerfreiheit das Ziel sein, sondern vielmehr bedarf es eines Bewusstseins dafür, dass Verstehen immer nur mit und durch Fehler möglich ist. ${ }^{41}$ Auch das Konzept des Forschenden Lernens bringt eine gewisse Fehlerfreundlichkeit mit sich und hält Momente der Überraschung und des Scheiterns als integralen Bestandteil von Forschung fest. Wie dies in Seminaren mit benoteten Prüfungsleistungen umsetzbar ist, bedarf weiteren Nachdenkens.

\subsubsection{Irritationen zulassen}

Eine postkoloniale Quellenanalyse hat immer das Interesse, vertraute Geschichtskonstruktionen zu irritieren. Die Universität ist ein Ort, an dem Irritationen und Beunruhigungen nicht nur zugelassen, sondern auch gefördert und produziert werden. Sie ist ein Ort, „an dem nichts außer Frage steht", um einen Ausdruck Derridas zu nutzen. ${ }^{42}$ Einsichten, Narrative und Zuschreibungen können und müssen immer wieder in Frage gestellt

\footnotetext{
40 SPIVAK, Can the Subaltern speak?, 32-41.

41 „I assume that the passing of a text into my grasp is a mis-take, of course. As we move towards the subaltern, we can only learn through mistakes." spivak, An Aesthetic Education, 28.

42 Zitiert nach: Susanne Arens/Susann Fegter et AL., Wenn Differenz in der Hochschullehre thematisch wird. Einführung in die Reflexion eines Handlungszusammenhangs, in: Paul Mecheril/Susanne Arens et al. (Hg.),
} 
werden. Auch die Fotografien aus dem Seminar sind Irritationen. Wenn sie als koloniale Quellen ernst genommen werden, verkomplizieren sie die Geschichtsschreibung.

Ein Junge mit einem Anzug neben einem Stuhl, dessen Einzelteile aus Europa nach Afrika geschickt wurden. Die nackten Füße sind eine vermeintlich exotische Irritation, die viele Interpretationen zulassen. Armut, Naturbezogenheit, Widerstand gegen europäische Kleiderordnungen, Schuhmangel in Afrika, Gefangenschaft, Ausdruck religiöser Ehrfurcht, Zeichen der Unschuld?

Das Buch in der Hand weist auf seine Lesefähigkeit hin. Lesen wurde im Protestantismus als ein wichtiger Bestandteil christlicher Religiosität wertgeschätzt. Das Buch zeugt von Erwartungen, denen der Junge gegenübersteht, aber auch von seinen Kompetenzen. Es erinnert an diejenigen, die ihn unterrichtet und die seinen Unterricht finanziert haben. Vielleicht ist es eine Bibel, vielleicht ein Gesangbuch. Zusammen mit dem Anzug und dem Stuhl ist es ein Ausdruck europäischer Kultur. Zugleich weist das eine Buch auf alle anderen Bücher hin, die in der Contact Zone der Missionsarbeit verteilt, übersetzt, angeeignet, umgedeutet und neuinterpretiert werden. ${ }^{43}$ Das eine Buch verweist auf all diejenigen Bücher, die der Junge noch lesen wird, oder noch lesen könnte, und wird so zum Symbol seiner vor ihm liegenden Zukunft.

Eine Mädchenschulklasse mit einer jungen Lehrerin. Alles in dem Foto ist auf die Kamera gerichtet: Die Sitzbänke, die Blickrichtung der Kinder, selbst die Tafel. Nur die Lehrerin sitzt schräg zur Klasse und ist deshalb im Profil zu sehen. Das Bild ist abgedruckt im Monatsblatt der NMG aus dem Jahr $1909 .{ }^{44}$ Das Bild ist in seiner ganzen Komposition auf diejenigen ausgerichtet, die es sich ansehen werden. Europäische Frauen, die einer beruflichen Tätigkeit nachgingen oder zuhause arbeiteten, Männer, die eine eigene Familie hatten oder alleinstehend waren, Kinder mit ambivalenter Einstellung zum Thema Schule, sie alle sahen sich das Foto an und ließen sich zu verschiedenen Gedanken inspirieren. Missionsfotografien und ihre Rezeption zeigen, wie sehr Mission wenn auch unterschiedlich erlebte, so doch gemeinsam geteilte Geschichte europäischer und afrikanischer Gesellschaften ist.

Differenz unter Bedingungen von Differenz. Zu Spannungsverhältnissen universitärer Lehre, Wiesbaden 2013, 7-28, 10.

43 Bhabha thematisiert das Buch im hybriden Raum kolonialer Macht in: BHAB HA, Location of Culture, $145^{-147}$.

44 Ella Lauenstein, Ein Vergleich der Schwesternarbeit in Keta und Lomé, in: MNdmg 70 (1909), 3-5. 


\section{Bibliographie}

Alsheimer, Rainer, Bilder erzählen Geschichten. Eine Fotoanthropologie der Norddeutschen Mission in Westafrika, Bremen 2010.

Arens, Susanne/Fegter, Susann et AL., Wenn Differenz in der Hochschullehre thematisch wird. Einführung in die Reflexion eines Handlungszusammenhangs, in: Paul Mecheril/Susanne Arens et Al. (Hg.), Differenz unter Bedingungen von Differenz. Zu Spannungsverhältnissen universitärer Lehre, Wiesbaden 2013, 7-28.

Bachmann-Medick, Doris, Cultural Turns. Neuorientierungen in den Kulturwissenschaften, Hamburg ${ }^{2} 2007$.

Becker, Judith/Stornig, Katharina (Hg.), Menschen - Bilder - Eine Welt. Ordnungen von Vielfalt in der religiösen Publizistik um 1900, VIEG 118, Göttingen 2018.

BECKER, JUdith (Hg.), European Missions in Contact Zones. Transformation through Interaction in a (Post-)Colonial World, VIEG.B 107, Göttingen 2015.

Becker, Judith (Hg.), Conversio im Wandel. Basler Missionare zwischen Europa und Südindien und die Ausbildung einer Kontaktreligiosität, 1834-1860, VIEg 238, Göttingen 2015 .

ВНАВ НА, НОмІ К., The location of culture, London/New York 1994.

В нав НА, Номі K., Die Verortung der Kultur, Tübingen 2000.

Bundesassistentenkonferenz (BAK), Forschendes Lernen - Wissenschaftliches Prüfen, Bonn 1970 (Neudruck Bielefeld 2009).

Chakrabarty, Dipesh, Europa provinzialisieren. Postkolonialität und die Kritik der Geschichte, in: Sebastian Conrad/Shalini Randeria (Hg.), Jenseits des Eurozentrismus. Postkoloniale Perspektive in den Geschichts- und Kulturwissenschaften, Frankfurt/New York 2002, 283-312.

ECKL, ANDREAS, Ora et labora. Katholische Missionsfotografie aus den afrikanischen Kolonien, in: Marianne Bechhaus-Gerst/Sunna Gieseke (Hg.), Koloniale und postkoloniale Konstruktionen von Afrika und Menschen afrikanischer Herkunft in der deutschen Alltagskultur, Frankfurt am Main 2006, 231-250.

ElmshäUser, KonRAD, Menschen in Afrika - aus dem Bildarchiv der Norddeutschen Mission, in: BrJ 85 (2006), 8-13.

Fichten, Wolfgang, Forschendes Lernen in der Lehrerbildung, in: Ulrike Eberhardt (Hg.), Neue Impulse in der Hochschuldidaktik, Wiesbaden 2010, 127-182.

Gruber, Judith, Wider die Entinnerung. Zur postkolonialen Kritik hegemonialer Wissenspolitiken in der Theologie, in: ANDreas NeHRIng/Simon WiesgickL (Hg.), Postkoloniale Theologien II. Perspektiven aus dem deutschsprachigen Raum, Stuttgart 2018, 23-37. 
Gutschera, Herbert/Thierfelder, Jörg, Weltmission in der Neuzeit, in: Jörg Thierfelder/Herbert Gutschera/Rainer Lachmann (Hg.), Kirchengeschichtliche Grundthemen. Historisch - systematisch - didaktisch, ThLL 3, Göttingen 42014, 252-270.

Heidemanns, Katja, Art. Mission/Missionarin (kath.), in: WfT (2002), 411-413.

Huber, Ludwig, Warum Forschendes Lernen nötig und möglich ist, in: LudWig Huber/Julia Hellmer/Friederike Schneider (Hg.), Forschendes Lernen im Studium. Aktuelle Konzepte und Erfahrungen, Bielefeld 2009, 9-36.

JÄGER, Jens, Fotografie und Geschichte, Frankfurt am Main/New York 2009 und Ulrike Pilarczyk/Ulrike Mietzner, Das reflektierte Bild. Die seriellikonografische Fotoanalyse in den Erziehungs- und Sozialwissenschaften, Bad Heilbrunn 2005.

Koschorke, Klaus (Hg.), Außereuropäische Christentumsgeschichte (Asien, Afrika, Lateinamerika), 1450-1990, Neukirchen-Vluyn ${ }^{4} 2012$.

LAUENSTE IN, Ella, Ein Vergleich der Schwesternarbeit in Keta und Lomé, in: MND MG 70 (1909), 3-5.

LudWIG, FrIEDER, Globale Christentumsgeschichte, in: VF 57/2 (2012), 106-117.

Morgan, David, The Sacred Gaze. Religious Visual Culture in Theory and Practice, Berkeley/Los Angeles 2005, 147-190.

Morton, Stephen, Gayatri Spivak. Ethics, Subalternity and the Critique of Postcolonial Reason, Key Contemporary Thinkers, Cambridge 2007.

Nausner, Michael, Die langen Schatten der Nofretete, in: Conc(D) 49/2 (2013), 200-209.

Pratt, Mary Louise, Arts of the Contact Zone, in: Profession 91 (1991), 33-40.

SAID, EDWARD, Orientalism, New York 1978.

SAID, EDWARD, Orientalismus, Frankfurt am Main 2009.

Schleiermacher, Friedrich Daniel Ernst, Gelegentliche Gedanken über Universitäten im deutschen Sinn (1808), in: DE Rs., Texte zur Pädagogik, Bd. 1, hg.v. MiCHAEL WinKLER, Frankfurt am Main 2000, 101-165.

Schmidt-Linsenhoff, Viktoria, Ästhetik der Differenz. Postkoloniale Perspektiven vom 16. bis 21. Jahrhundert, 2 Bde., Marburg 2010.

Sommer, Wolfgang/Detlef Klahr/Marcel Nieden, Kirchengeschichtliches Repetitorium. Zwanzig Grundkapitel der Kirchen-, Dogmen- und Theologiegeschichte, Göttingen u.a. ${ }^{5} \mathbf{2 0 1 2}$.

Spivak, Gayatri Chakravorty, The Post-Colonial Critic. Interviews, Strategies, Dialogues, hg.v. SARAH Harasym, New York/London 1990.

Spivak, Gayatri Chakravorty, Outside in the Teaching Machine, London/New York 1993 . 
Spivak, Gayatri Chakravorty, A Critique of Post-Colonial Reason. Toward a History of the Vanishing Present, Cambridge (MA)/London 1999.

Spivak, Gayatri Chakravorty, Can the Subaltern Speak? Postkolonialität und subalterne Artikulation. Übers. Alexander Joskowicz, Stefan Nowotny, Einl. Hito Steye RL, Wien 2008 (erstmals erschienen 1988).

Spivak, Gayatri Chakravorty, An Aesthetic Education in the Era of Globalization, Cambridge (MA) 2012.

Spivak, Gayatri Chakravorty, „Why Study the Past?“, in: MLQ 73.1 (2012), 1-12.

Spivak, Gayatri Chakravorty, Occupy Education. An Interview with Gayatri Chakravorty Spivak by Rahul K. Gairola, https://politicsandculture.org/2012/og/25/ occupy-education-an-interview-with-gayatri-chakravorty-spivak/ [Zugriff: 30.9.2017].

Stornig, Katharina, Vielfache Bedeutungen. Missionsfotografie zwischen Neuguinea und Europa, 1899-ca. 1930, in: Österreichische Zeitschrift für Geschichtswissenschaften 24/2 (2013), 114-139.

Stornig, Katharina, Authentifizierung kultureller Begegnungen durch Fotografie. Über die Verwendung von Fotos als Spuren in der transnationalen Spendenwerbung im 19. Jahrhundert, in: Saec. 66/2 (2016), 207-228.

Thоmpson, T. Jack, Light on Darkness? Missionary Photography of Africa in the Nineteenth and Early Twentieth Century, Grand Rapids (MI)/Cambridge (UK) 2012.

WissenschaftsRat, Empfehlungen zur künftigen Struktur der Lehrerausbildung, Berlin 2001. 